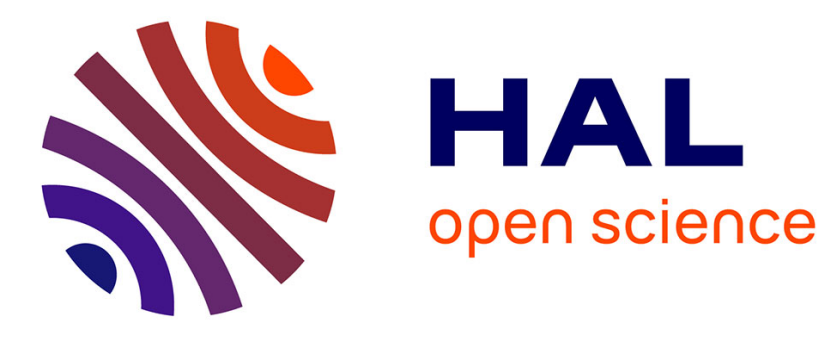

\title{
An extension of component-trees to partial orders
}

\author{
Nicolas Passat, Benoît Naegel
}

\section{To cite this version:}

Nicolas Passat, Benoit Naegel. An extension of component-trees to partial orders. International Conference on Image Processing (ICIP), 2009, Cairo, Egypt. pp.3981-3984, 10.1109/ICIP.2009.5413807 . hal-01695031

\section{HAL Id: hal-01695031 \\ https://hal.univ-reims.fr/hal-01695031}

Submitted on 3 Mar 2018

HAL is a multi-disciplinary open access archive for the deposit and dissemination of scientific research documents, whether they are published or not. The documents may come from teaching and research institutions in France or abroad, or from public or private research centers.
L'archive ouverte pluridisciplinaire HAL, est destinée au dépôt et à la diffusion de documents scientifiques de niveau recherche, publiés ou non, émanant des établissements d'enseignement et de recherche français ou étrangers, des laboratoires publics ou privés. 


\section{AN EXTENSION OF COMPONENT-TREES TO PARTIAL ORDERS}

Nicolas Passat

\author{
LSIIT, UMR CNRS 7005 \\ Strasbourg University \\ France
}

\author{
Benoît Naegel \\ LORIA, UMR CNRS 7503 \\ Nancy 1 University \\ France
}

\begin{abstract}
Component-trees provide efficient ways to define filteringbased procedures on grey-level images. We propose an extension of the notion of component-trees to the case of "non grey-level" images (i.e. images taking their values in partially-ordered sets) including in particular - but not exclusively - colour images. Experiments performed on such images emphasise the interest of the approach.
\end{abstract}

Index Terms - Mathematical morphology, componenttrees, filtering, partial orders, colour imaging

\section{INTRODUCTION}

The component-tree models some structural characteristics of an image by considering its "binary slices" obtained by threshold operations at successive levels. It has been defined in the theoretical framework of mathematical morphology and involved, in particular, in the development of morphological operators $[1,2]$.

Thanks to efforts devoted to the efficient computation of component-trees [2, 3], or their use in complex knowledge handling procedures, they have been considered for the design of various kinds of grey-level image processing methods, including image and video segmentation [2, 4], image registration [5] or image compression [2].

We propose to explore new ways for the use of componenttrees by not only considering images taking their values in completely-ordered sets (i.e. "grey-level" images), but more generally in partially-ordered ones, such as colour images, for instance. A data structure and some strategies for filtering such images are described. They are, in particular, compliant with the classical approaches proposed in the grey-level case. Experimental results enable to illustrate the soundness and usefulness of the approach.

The article is organised as follows. In Sec. 2, theoretical background notions are provided. In Sec. 3, the definition of the component-tree, and classical ways for using it in filtering procedures are recalled. In Sec. 4, the proposed extension of component-trees to images taking their values in partiallyordered sets is described. Experimental results and perspectives will be found in Sec. 5 .

\section{THEORETICAL BACKGROUND}

Let $n \in \mathbb{N}^{*}$. Let $E \subset \mathbb{Z}^{n}$ (generally, $E=\prod_{i=1}^{n}\left[0, d_{i}-1\right]$ with $\left.\left(d_{i}\right)_{i=1}^{n} \in\left(\mathbb{N}^{*}\right)^{n}\right)$. Let $V$ be a finite set. Let $\leq$ be an order relation on $V$. We suppose that there exists an infimum, noted $\perp$, for $(V, \leq)$. Let $I: E \rightarrow V$ (we also note $I \in V^{E}$ ), $I$ is a (discrete) image, taking its values in $V$. If $(V, \leq)$ is a completely-ordered (resp. partially-ordered) set, then we say that $I$ is a grey-level image (resp. a label image).

Let $x, y \in E$. We say that $x$ and $y$ are adjacent if $d(x, y) \leq \epsilon$ for a well-chosen distance $d(.,$.$) on E$ and a nonnegative value $\epsilon$ (with $\epsilon=1$ and $d(.,)=.\|.-\|_{1}$ (resp. $\left.d(.,)=.\|.-.\|_{\infty}\right)$, we retrieve the classical $(2 n)$-adjacency (resp. $\left(3^{n}-1\right)$-adjacency) associated to $\left.\mathbb{Z}^{n}\right)$.

Let $X \subseteq E$. Let $x, y \in X$. We say that $x$ and $y$ are connected (in $\bar{X}$ ), and we note $x \sim y$, if there exists a sequence $\left(x_{k}\right)_{k=1}^{l}(l \geq 1)$ of elements of $X$ such that $x_{1}=x, x_{l}=y$ and $x_{k}, x_{k+1}$ are adjacent for all $k \in[1, l-1]$. Note that $\sim$ is an equivalence relation on $X$. The connected components of $X$ are the equivalence classes of $X$ w.r.t. $\sim$, i.e. the elements of the quotient set $X / \sim($ noted $\mathcal{C}[X]$ in the sequel).

Let $v \in V$. Let $X_{v}: V^{E} \rightarrow \mathcal{P}(E)$ be the threshold operator defined by $X_{v}(I)=\{x \in E \mid v \leq I(x)\}$ for all $I \in V^{E}$

Let $v \in V$ and $X \subseteq E$. We define the cylinder function $C_{X, v}: E \rightarrow V$ by $C_{X, v}(x)=v$ if $x \in X$ and $\perp$ otherwise. Based on this definition, a discrete image $I \in V^{E}$ can be expressed as $I=\bigvee_{v \in V} C_{X_{v}(I), v}=$ $\bigvee_{v \in V} \bigvee_{X \in \mathcal{C}\left[X_{v}(I)\right]} C_{X, v}$, where $\bigvee$ is the pointwise supremum of a set of functions, i.e. $I(x)=\sup _{v \in V}\left\{C_{X_{v}(I), v}(x)\right\}$ $\left(=\max _{v \in V}\left\{C_{X_{v}(I), v}(x)\right\}\right.$ as $V$ is finite).

Let $\mathcal{K}=\bigcup_{v \in V}\left(\mathcal{C}\left[X_{v}(I)\right] \times\{v\}\right)$. Let $\subseteq_{\mathcal{K}}$ be the relation defined by $\left(X, v_{X}\right) \subseteq \subseteq_{\mathcal{K}}\left(Y, v_{Y}\right)$ if $X \subset Y$ or $(X=Y$ and $\left.v_{Y} \leq v_{X}\right)$. The inclusion relation $\subseteq \mathcal{K}$ is a partial order on $\mathcal{K}$. By abuse of notation, in the sequel, we will sometimes note $X \in \mathcal{K}$ for $\left(X, v_{X}\right) \in \mathcal{K}$, and $\subseteq$ for $\subseteq \mathcal{K}$.

\section{COMPONENT-TREES}

In this section, we assume that $(V, \leq)$ is a completely-ordered set. In particular, we identify $(V, \leq)$ and $([0,|V|-1], \leq)$. 


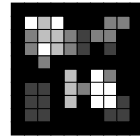

(a)

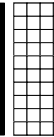

(b)

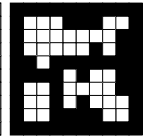

(c)

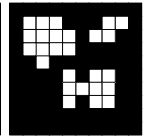

(d)

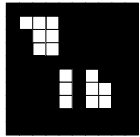

(e)

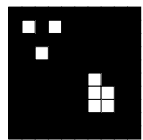

(f)

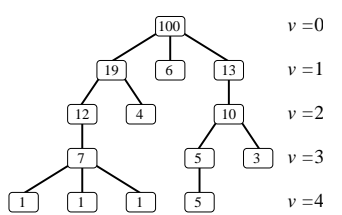

(g)

Fig. 1. (a) A grey-level image $I:[0,9]^{2} \rightarrow[0,4]$. (bf) Threshold sets $X_{v}(I)$ for $v$ from 0 (b) to 4 (f). (g) The component-tree of $I$; its successive levels correspond to increasing threshold values $v$. (h) The same tree, enriched by an attribute (the size of the connected component of each node).

Let $v_{1} \leq v_{2} \in V$. Let $B_{1}, B_{2} \subseteq E$ be the binary images defined by $B_{k}=X_{v_{k}}(I)$ for $k \in\{1,2\}$. Let $C_{2} \in \mathcal{C}\left[B_{2}\right]$ be a connected component of $B_{2}$. Then, there exists a (unique) connected component $C_{1} \in \mathcal{C}\left[B_{1}\right]$ of $B_{1}$ such that $C_{2} \subseteq C_{1}$ (see Fig. 1 (b-f) for an illustration of this assertion). Note that, in particular, we necessarily have $B_{2} \subseteq B_{1}$.

Based on these considerations, it easily derives that the Hasse diagram of the partially-ordered set $(\mathcal{K}, \subseteq)$ is a tree (i.e. a connected acyclic graph), the "root" of which is the supremum $\sup (\mathcal{K}, \subseteq)=X_{\perp}(I)=E$. This tree is called the component-tree of $I$ (see Fig. $1(\mathrm{~g})$ ).

Using the above definitions and notations, the componenttree - as a data structure - can be described as follows.

Definition Let $I \in V^{E}$ be a grey-level image. The componenttree of $I$ is the rooted tree $(\mathcal{K}, L, R)$ defined by

$$
\begin{aligned}
& R=E=\sup (\mathcal{K}, \subseteq), \\
& L=\left\{(X, Y) \in \mathcal{C}\left[X_{v}(I)\right] \times \mathcal{C}\left[X_{v+1}(I)\right] \mid Y \subseteq X\right\}
\end{aligned}
$$

The elements $\mathcal{K}, R$ and $L$ are the set of the nodes, the root and the set of the edges of the tree, respectively (in the sequel, we will use the usual terminology associated to trees). In Fig. $1(\mathrm{~g}), \mathcal{K}$ is the set of all white rectangles $(R$ is the one located at the highest level) and $L$ is visualised by the set of all black edges (linking the elements of each pair).

Component-trees enable the storage - at each node - of elements of information, also called attributes, related to the binary connected component associated to the node. For instance, in Fig. 1(h), the size (i.e. the cardinal) of the connected components has been added at their corresponding nodes ${ }^{1}$.

Component-trees, equipped with attributes related to their image, are generally considered for filtering operations con-

\footnotetext{
${ }^{1}$ Here, the attribute is a single numerical value. It is however possible to consider any kind of (quantitative/qualitative and scalar/vectorial) attributes, provided they can be conveniently formalised.
}

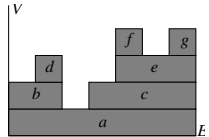

(a)

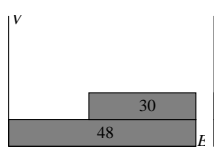

(c) (b)

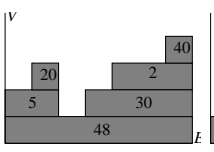

(d)

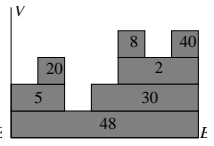

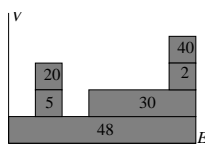

(e)

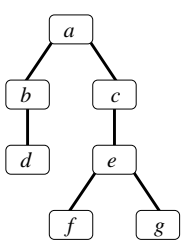

(f)
Fig. 2. (a) A 1-D grey-level image $I$ and (f) its componenttree. (b) (Fictive) non-increasing attributes associated to the nodes of $I$. (c-e) Filtering results $I_{\rho}$ obtained by processing the component-tree w.r.t. a predicate $\rho$ true if and only if the attribute of the node is higher than 15: (c) Min, (d) Max and (e) Direct policies.

sisting in "pruning" the branches of the tree, based on a chosen predicate $\rho$ on $\mathcal{K}$, indicating if the attribute of a node satisfies a given criterion. In case of non-increasing criteria, various pruning policies can be considered (see [6]):

(i) Min: A node $N \in \mathcal{K}$ is removed if $\neg \rho(N)$ or the parent node $P \in \mathcal{K}$ of $N$ has been removed;

(ii) Max: A node $N \in \mathcal{K}$ is removed if $\neg \rho(N)$ and all its children $C \in \mathcal{K}$ have been removed;

(iii) Direct: A node $N \in \mathcal{K}$ is removed if $\neg \rho(N)$.

Once the set $\mathcal{K}$ has been reduced to a set $\mathcal{K}_{\rho} \subseteq \mathcal{K}$ according to one of these three policies, a reconstructed image $I_{\rho}$ of $I$ can be defined as $I_{\rho}=\bigvee_{\left(X, v_{X}\right) \in \mathcal{K}_{\rho}} C_{X, v_{X}}$. Filtering and reconstruction results of these strategies are illustrated on a 1-D example in Fig. 2.

\section{EXTENSION TO PARTIAL ORDERS}

In this section, we now assume that $(V, \leq)$ is a partiallyordered set and that $(V, \leq)$ has an infimum, noted $\perp$ (otherwise we enrich $(V, \leq)$ with such an infimum).

Let $I \in V^{E}$ be a label image. Let $v \in V$. Let $B \subseteq E$ be the binary image defined by $B=X_{v}(I)$. Let $C \in \overline{\mathcal{C}}[B]$ be a connected component of $B$. By opposition to the case of completely-ordered sets (Sec. 3), there may exist several values $v_{i} \leq v(i \in \mathbb{N})$, such that $C \subseteq C_{i} \in \mathcal{C}\left[X_{v_{i}}(I)\right]$, and $v_{i}$ is maximal w.r.t. $\leq$ for these properties.

As in Sec. 3, the relation $\subseteq$ is still a partial order on $\mathcal{K}$, but the Hasse diagram of the partially-ordered set $(\mathcal{K}, \subseteq)$ is not necessarily a tree. This derives from the fact that - using tree terminology - a node of the diagram can be the child of several parent nodes (and not only one). Note that such a diagram (called component-graph in the sequel) has however a supremal element (which can be assimilated to its "root"), namely $X_{\perp}(I)=E$, while it can possibly have several minimal elements. 


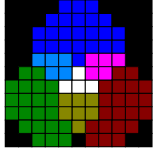

(a)

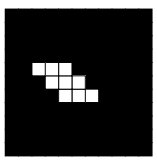

(e)

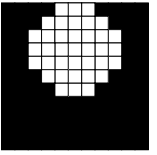

(b)

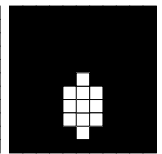

(f)

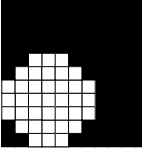

(c)

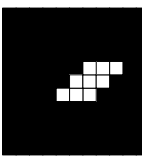

(g)

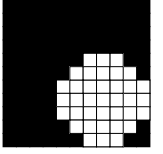

(d)

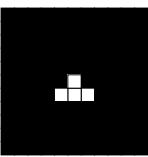

(h)

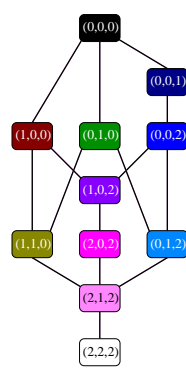

(i)
Fig. 3. (a) A label image $I:[0,10]^{2} \rightarrow V$ (where $V \subset[0,2]^{3}$ is composed of 11 distinct values depicted in (i)) visualised as an RGB image. (b-h) Some threshold images $X_{v}(I)$ (from (b) to (h), $v=(0,0,2),(0,1,0),(1,0,0),(0,1,2),(1,1,0)$, $(2,0,2),(2,2,2)$. (i) The component graph of $I$.

\subsection{Component-graphs}

Let $I \in V^{E}$ be a label image. The component-graph of $I$ is the "rooted" oriented graph $(\mathcal{K}, L, R)$ defined such that $(\mathcal{K}, L)$ is the Hasse diagram of the partially-ordered set $(\mathcal{K}, \subseteq$ ), and $R=\sup (\mathcal{K}, \subseteq)=X_{\perp}(I)=E$. Keeping the same terminology as previously, $\mathcal{K}, R$ and $L$ are the set of the nodes, the root and the set of the edges of the graph, respectively.

Fig. 3 illustrates a label image $I$ (a), some of its threshold images (b-h), and its component graph (i): $\mathcal{K}$ is the set of all rectangles ( $R$ is the black one) and $L$ is visualised by the set of all black edges (linking the elements of each pair).

Similarly to component-trees, attributes can be stored at each node of a component-graph. The preservation/removal of nodes of the graph w.r.t. such attributes can then also lead to filtering strategies. However, the less restrictive properties of component-graphs (by opposition to those of componenttrees) do not enable to straightforwardly apply the filtering strategies designed for component-trees, described in Sec. 3. To tackle this problem, we propose hereafter a two step filtering procedure.

\subsection{Filtering strategy}

Let $I \in V^{E}$ be a label image and $(\mathcal{K}, L, R)$ be its componentgraph (the image $I$ of Fig. 3(a) will be considered for illustration). Let $\rho$ be a predicate on $\mathcal{K}$. The following strategy is devoted to the filtering of $I$ by "pruning" its component-graph w.r.t. $\rho$.

\subsubsection{Step 1: Node selection}

Let $\mathcal{K}^{\prime}=\mathcal{K} \backslash\{R\}$. In case of non-increasing criteria (notice that with increasing ones, all policies are equivalent), the following policies can be considered to select a subset $\mathcal{K}_{\rho} \subseteq \mathcal{K}$ of nodes to be preserved in the component-graph:

(i) $\operatorname{Min}_{1}$ : A node $N \in \mathcal{K}^{\prime}$ is removed if $\neg \rho(N)$ or at least one

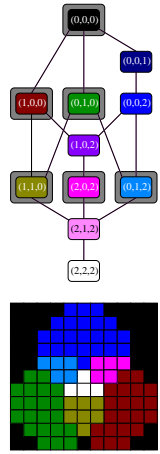

(a)

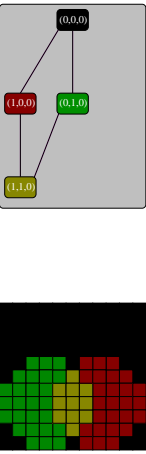

(b)
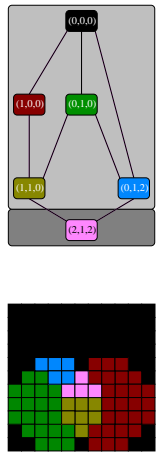

(c)
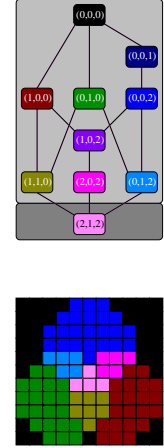

(d)

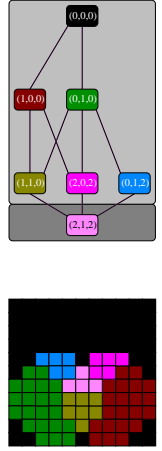

(e)
Fig. 4. (a) $I$ and its component-graph, with nodes verifying a given predicate $\rho$ (grey-background). (b-e) Light-grey background: graph $\left(\mathcal{K}_{\rho}, L_{\rho}\right)$ obtained with the $\operatorname{Min}_{1}$ (b), $\operatorname{Min}_{2}$ (c), $\operatorname{Max}$ (d), and Direct (e) policies; light/dark-grey background: corrected graph $\left(\mathcal{K}_{\rho}^{c}, L_{\rho}^{c}\right)$.

of the parent node $P \in \mathcal{K}$ of $N$ has been removed;

(ii) Min $_{2}$ : A node $N \in \mathcal{K}^{\prime}$ is removed if $\neg \rho(N)$ or all the parent nodes $P \in \mathcal{K}$ of $N$ have been removed;

(iii) Max: A node $N \in \mathcal{K}$ is removed if $\neg \rho(N)$ and all its children $C \in \mathcal{K}^{\prime}$ have been removed;

(iv) Direct: A node $N \in \mathcal{K}$ is removed if $\neg \rho(N)$.

Note that with the $\operatorname{Min}_{1}$ and $\operatorname{Min}_{2}$ (resp. Max) policy(ies), $\mathcal{K}_{\rho}$ has to be computed in a top-down (resp. bottom-up) fashion. Moreover, with the first three policies, we should assume that $R \in \mathcal{K}_{\rho}$ (otherwise, we would obtain a - useless - trivial set $K_{\rho}=\emptyset$ ). More formally, with the $\operatorname{Min}_{1}$ (3), $\operatorname{Min}_{2}$ (4), Max (5), and Direct (6) policies, we have (recursively)

$$
\begin{aligned}
& \mathcal{K}_{\rho}=\{R\} \cup\left\{N \in \mathcal{K}^{\prime} \mid \rho(N) \wedge \forall(P, N) \in L, P \in \mathcal{K}_{\rho}\right\}, \text { (3) } \\
& \mathcal{K}_{\rho}=\{R\} \cup\left\{N \in \mathcal{K}^{\prime} \mid \rho(N) \wedge \exists(P, N) \in L, P \in \mathcal{K}_{\rho}\right\}, \text { (4) } \\
& \mathcal{K}_{\rho}=\{R\} \cup\left\{N \in \mathcal{K}^{\prime} \mid \rho(N) \vee \exists(N, C) \in L, C \in \mathcal{K}_{\rho}\right\},(5) \\
& \mathcal{K}_{\rho}=\{N \in \mathcal{K} \mid \rho(N)\} .
\end{aligned}
$$

Once $\mathcal{K}_{\rho}$ has been computed, a new set of edges $L_{\rho}$ can straightforwardly be defined by building the Hasse diagram of $\left(\mathcal{K}_{\rho}, \subseteq\right)$. Examples of graphs $\left(\mathcal{K}_{\rho}, L_{\rho}\right)$ obtained with these different policies are illustrated in Fig. 4.

\subsubsection{Step 2: Coherence recovery}

By considering the $\operatorname{Min}_{1}$ and $\operatorname{Min}_{2}$ (resp. Max, Direct) policy(ies) proposed above, in the case of a completely-ordered set, we obviously retrieve the "classical" Min (resp. Max, Direct) policy defined in Sec. 3. The proposed approach is then compliant with the case of component-trees.

However, in the considered case of a partially-ordered set $(V, \leq)$, it may sometimes be impossible to reconstruct an image $I_{\rho}=\bigvee_{\left(X, v_{X}\right) \in \mathcal{K}_{\rho}} C_{X, v_{X}}$ defined by $I_{\rho}(x)=$ 
$\max _{\left(X, v_{X}\right) \in \mathcal{K}_{\rho}}\left\{C_{X, v_{X}}(x)\right\}$ for all $x \in E$, since such a maximum may be undefined, due to the partiality of $(V, \leq)$. As an example, in Fig. 4, the graph of (b) directly leads to a "correct" image $I_{\rho}$, while it is not the case for those of (c-e) since the lowest nodes of the graph correspond to intersecting connected components associated to non-comparable values.

In order to deal with this issue, a coherence recovery procedure has to be proposed to define unambiguously $\max _{\left(X, v_{X}\right) \in \mathcal{K}_{\rho}}\left\{C_{X, v_{X}}\right\}$ for all $x \in E$. This procedure consists in adding to the current graph $\left(\mathcal{K}_{\rho}, L_{\rho}\right)$ a set of nodes (actually removed during the previous step) and their associated edges, such that the resulting corrected graph $\left(\mathcal{K}_{\rho}^{c}, L_{\rho}^{c}\right)$ enables the generation of a well-defined image. Practically, this can be done by computing iteratively - and until stability - $\mathcal{K}_{\rho}^{c}$ (initialised to $\mathcal{K}_{\rho}$ ) as follows. Choose a point $x \in E$ and two nodes $N_{i}=\left(X_{i}, v_{X_{i}}\right) \in \mathcal{K}_{\rho}^{c}(i \in\{1,2\})$ (with $N_{1} \nsubseteq N_{2}, N_{2} \nsubseteq N_{1}$ ) such that $x \in X_{1} \cap X_{2}$ and $\forall N=\left(X, v_{X}\right) \in \mathcal{K}_{\rho}^{c} \backslash\left\{N_{i}\right\}, x \in X \Rightarrow\left(N \nsubseteq N_{i}\right)$. Then, there exists $N=\left(X, v_{X}\right) \in \mathcal{K} \backslash \mathcal{K}_{\rho}^{c}$ such that $x \in X \subseteq X_{1} \cap X_{2}$ and $v_{X_{1}}, v_{X_{2}} \leq v_{X}$. Choose such a node $N$ and set $\mathcal{K}_{\rho}^{c}=\mathcal{K}_{\rho}^{c} \cup\{N\}$. Once $\mathcal{K}_{\rho}^{c}$ has been computed, $L_{\rho}^{c}$ is obtained by computing the Hasse diagram of $\left(\mathcal{K}_{\rho}^{c}, \subseteq\right)$.

Note that the element $N$ may be non-unique at a given iteration, leading to a possibly non-deterministic process, and thus various results (in the case where $(V, \leq)$ is a lattice, some deterministic strategies can however be developed; this will be considered in further works). Examples of corrected graphs and their associated image are available in Fig. 4(c-e).

\section{EXPERIMENTAL RESULTS AND PERSPECTIVES}

We have introduced an extension of the notion of componenttrees (namely the component-graphs) to the case of partiallyordered sets, enabling in particular to deal with colour images.

To illustrate the soundness of this new concept, a denoising example is proposed on a colour image (see Fig. 5). Considering a predicate $\rho$ based on the size of the connected components of the nodes, a two-step component-graph filtering procedure (with the $\leq$ and $\geq$ partial orders on the RGB space, successively) is applied with a Direct policy on an image (a) corrupted with $10 \%$ (b) and $15 \%$ (c) random noise. It can be observed that the result images $(\mathrm{e}, \mathrm{f})$ present visual qualities quite similar to the original one $(\mathrm{a}, \mathrm{d})$.

This simple example (presented here due to space limitations) is - of course - not the only nor the main application field of component-graph approaches: more sophisticated ones (applied on both real colour images and "synthetic" ones [7] obtained from hierarchical classification processes, for instance) will be presented in further works. From a methodological point of view, we will also develop this preliminary study by extending classical concepts of the component-trees such that the Subtractive or Viterbi policies, and by developing alternative coherence recovery strategies.

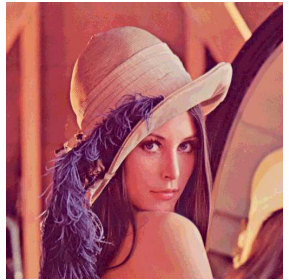

(a)

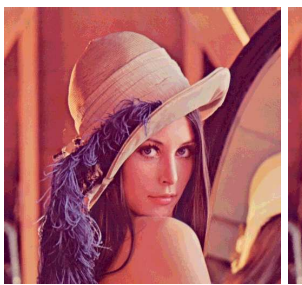

(d)

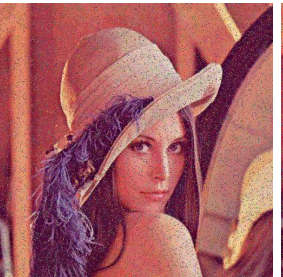

(b)

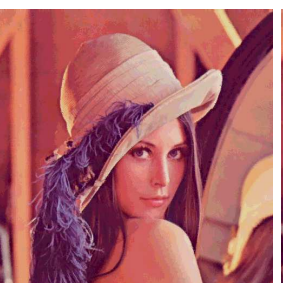

(e)

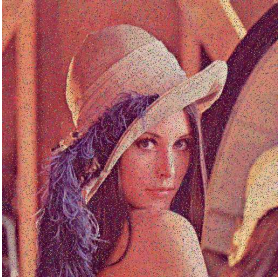

(c)

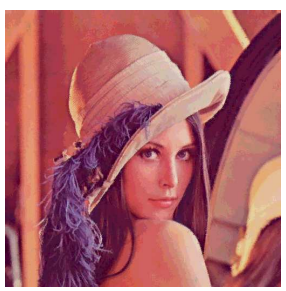

(f)
Fig. 5. (a,d) Original image (Lenna, (c)Playboy). (b,c) Noisy image (10\% and $15 \%$ random noise corruption, respectively). (e-f) Filtered images obtained from $(b, c)$ by removing the connected component of size smaller than 10 pixels.

\section{REFERENCES}

[1] E.J. Breen and R. Jones, "Attribute openings, thinnings, and granulometries," Computer Vision and Image Understanding, vol. 64, no. 3, pp. 377-389, 1996.

[2] P. Salembier, A. Oliveras, and L. Garrido, "Anti-extensive connected operators for image and sequence processing," IEEE Transactions on Image Processing, vol. 7, no. 4, pp. 555-570, 1998.

[3] L. Najman and M. Couprie, "Building the component tree in quasi-linear time," IEEE Transactions on Image Processing, vol. 15, no. 11, pp. 3531-3539, 2006.

[4] R. Jones, "Connected filtering and segmentation using component trees," Computer Vision and Image Understanding, vol. 75, no. 3, pp. 215-228, 1999.

[5] J. Mattes, M. Richard, and J. Demongeot, "Tree representation for image matching and object recognition," in DGCI. 1999, vol. 1568 of LNCS, pp. 298-312, Springer.

[6] E.R. Urbach, J.B.T.M. Roerdink, and M.H.F. Wilkinson, "Connected shape-size pattern spectra for rotation and scale-invariant classification of gray-scale images," IEEE Transactions on Pattern Analysis and Machine Intelligence, vol. 29, no. 2, pp. 272-285, 2007.

[7] C. Ronse and V. Agnus, "Morphology on label images: flat-type operators and connections," Journal of Mathematical Imaging and Vision, vol. 22, no. 2-3, pp. 283307, 2005. 